\title{
$f$-Statistical convergence of order $\alpha$ and strong Cesàro summability of order $\alpha$ with respect to a modulus
}

\author{
Vinod K Bhardwaj ${ }^{1 *}$ and Shweta Dhawan ${ }^{2}$
}

"Correspondence:
vinodk_bhj@rediffmail.com
${ }^{1}$ Department of Mathematics,
Kurukshetra University, Kurukshetra,
136119 , India
Full list of author information is
available at the end of the article

available at the end of the article

\begin{abstract}
In this paper, following a very recent and new approach of Aizpuru et al. (Quaest. Math. 37:525-530, 2014), we further generalize a concept of $\alpha$-density to that of $f_{\alpha}$-density, where $f$ is an unbounded modulus and $0<\alpha \leq 1$. As a consequence, we obtain a new nonmatrix convergence method, namely $f$-statistical convergence of order $\alpha$ or $S_{\alpha}^{f}$-convergence, which is intermediate between the ordinary convergence and the statistical convergence of order $\alpha$. We also introduce a new concept of strong Cesàro summability of order $\alpha$ with respect to a modulus function $f$, and finally we investigate the relationship between the set $S_{\alpha}^{f}$ of all $f$-statistically convergent sequences of order $\alpha$ and the set $w_{\alpha}^{f}$ of all strongly Cesàro summable sequences of order $\alpha$ with respect to $f$.
\end{abstract}

MSC: 40A35; 40C05; 46A45

Keywords: modulus function; density; statistical convergence; strong Cesàro summability

\section{Introduction and historical background}

The credit of introducing the idea of statistical convergence, which is in fact a generalization of the usual notion of convergence, goes to Zygmund [1]. Formally the concept of statistical convergence was introduced by Steinhaus [2] and Fast [3] and later reintroduced by Schoenberg [4]. Statistical convergence also arises as an example of 'convergence in density' as introduced by Buck in [5].

Statistical convergence has emerged as one of the most active areas of research in summability theory after the pioneering works of Šalát [6] and Fridy [7]. Later on statistical convergence was further investigated from the sequence space point of view and linked with summability theory by Connor [8], Çinar et al. [9], Et et al. [10], Savas [11], Sengül and Et [12], and many others.

Let $\mathbb{N}$ denote the set of all natural numbers. The number sequence $x=\left(x_{k}\right)$ is said to be statistically convergent to the number $l$ if for each $\varepsilon>0$ the set $\left\{k \in \mathbb{N}:\left|x_{k}-l\right| \geq \varepsilon\right\}$ has natural density zero, where the natural density of a subset $K \subset \mathbb{N}$ (see [13], Chapter 11) is defined by

$$
d(K)=\lim _{n \rightarrow \infty} \frac{1}{n}|\{k \leq n: k \in K\}|,
$$

(C) 2015 Bhardwaj and Dhawan. This article is distributed under the terms of the Creative Commons Attribution 4.0 International License (http://creativecommons.org/licenses/by/4.0/), which permits unrestricted use, distribution, and reproduction in any medium, provided you give appropriate credit to the original author(s) and the source, provide a link to the Creative Commons license, and indicate if changes were made. 
where $|\{k \leq n: k \in K\}|$ denotes the number of elements of $K$ not exceeding $n$. Obviously we have $d(K)=0$ provided that $K$ is a finite set of positive integers. If a sequence is statistically convergent to $l$, then we write it as $S$ - $\lim x_{k}=l$. The set of all statistically convergent sequences is denoted by $S$.

The idea of a modulus function was structured by Nakano [14] in 1953. Following Ruckle [15] and Maddox [16], we recall that a modulus $f$ is a function from $[0, \infty)$ to $[0, \infty)$ such that (i) $f(x)=0$ if and only if $x=0$, (ii) $f(x+y) \leq f(x)+f(y)$ for $x \geq 0, y \geq 0$, (iii) $f$ is increasing, (iv) $f$ is continuous from the right at 0 . Hence $f$ must be continuous everywhere on $[0, \infty)$. A modulus may be unbounded or bounded. For example, $f(x)=x^{p}$ where $0<$ $p \leq 1$, is unbounded, but $f(x)=\frac{x}{1+x}$ is bounded.

Connor [17], Ghosh and Srivastava [18], Bhardwaj and Singh [19-21], Çolak [22], Altin and Et [23] and some others have used a modulus function to construct some sequence spaces.

In the year 2014, Aizpuru et al. [24] defined a new concept of density with the help of an unbounded modulus function and, as a consequence, they obtained a new concept of nonmatrix convergence which is intermediate between the ordinary convergence and the statistical convergence, and agrees with the statistical convergence when the modulus function is the identity mapping.

Quite recently, Bhardwaj et al. [25] have introduced and studied a new concept of $f$-statistical boundedness by using the approach of Aizpuru et al. [24]. It is shown that the concept of $f$-statistical boundedness is intermediate between the ordinary boundedness and the statistical boundedness. It is also proved that bounded sequences are precisely those sequences which are $f$-statistically bounded for every unbounded modulus $f$.

We now recall some definitions that will be needed in the sequel.

Definition 1.1 [24] Let $f$ be an unbounded modulus function. The $f$-density of a set $A \subset \mathbb{N}$ is defined by

$$
d^{f}(A)=\lim _{n \rightarrow \infty} \frac{f(|\{k \leq n: k \in A\}|)}{f(n)}
$$

in case this limit exists.

Remark 1.2 The concept of $f$-density reduces to that of natural density when $f(x)=x$. In case of natural density, it is well known that $d(A)+d(\mathbb{N}-A)=1$. But this result remains no longer true in case of $f$-density, i.e., $d^{f}(A)+d^{f}(\mathbb{N}-A)=1$ does not hold, in general. For example, if we take $f(x)=\log (x+1)$ and $A=\{2 n: n \in \mathbb{N}\}$, then $d^{f}(A)=d^{f}(\mathbb{N}-A)=1$. However, in case of $f$-density, we can assert that if $d^{f}(A)=0$ then $d^{f}(\mathbb{N}-A)=1$. As in the case of natural density, finite sets also have zero $f$-density and so for any finite set $A$, $d^{f}(A)+d^{f}(\mathbb{N}-A)=1$.

Note that Aizpuru et al. [24] have used the notation $d_{f}(A)$ to denote the $f$-density of $A$.

Remark 1.3 For any unbounded modulus $f$ and $A \subset \mathbb{N}, d^{f}(A)=0$ implies that $d(A)=0$. But converse need not be true in the sense that a set having zero natural density may have non-zero $f$-density with respect to some unbounded modulus $f$. For example, if we take $f(x)=\log (x+1)$ and $A=\{1,4,9, \ldots\}$, then $d(A)=0$ but $d^{f}(A)=1 / 2$. However, in view of Remark 1.2, $d(A)=0$ implies $d^{f}(A)=0$ is always true in case of any finite set $A \subset \mathbb{N}$, irrespective of the choice of unbounded modulus $f$. 
Definition 1.4 [24] Let $f$ be an unbounded modulus function. A number sequence $x=$ $\left(x_{k}\right)$ is said to be $f$-statistically convergent to $l$, or $S^{f}$-convergent to $l$, if for each $\varepsilon>0$,

$$
\begin{aligned}
& d^{f}\left(\left\{k \in \mathbb{N}:\left|x_{k}-l\right| \geq \varepsilon\right\}\right)=0, \\
& \quad \text { i.e., } \lim _{n \rightarrow \infty} \frac{1}{f(n)} f\left(\left|\left\{k \leq n:\left|x_{k}-l\right| \geq \varepsilon\right\}\right|\right)=0,
\end{aligned}
$$

and we write it as $S^{f}-\lim x_{k}=l$. The set of all $f$-statistically convergent sequences is denoted by $S^{f}$.

In view of Definition 1.4 and Remark 1.3 , it follows that every $f$-statistically convergent sequence is statistically convergent, but a statistically convergent sequence need not be $f$-statistically convergent for every unbounded modulus $f$.

Statistical convergence of order $\alpha(0<\alpha \leq 1)$ was introduced by Çolak [26], and also independently by Bhunia et al. [27], using the notion of natural density of order $\alpha$ (where $n$ is replaced by $n^{\alpha}$ in the denominator in the definition of natural density). It was observed in $[26,27]$ that the behavior of this new kind of convergence was not exactly parallel to that of statistical convergence. For a detailed account of many more interesting investigations concerning statistical convergence of order $\alpha$, one may refer to [9, 10, 28-31] and [12].

Definition 1.5 [26] Let $\alpha$ be any real number such that $0<\alpha \leq 1$. The $\alpha$-density of a set $A \subset \mathbb{N}$ is defined by

$$
d_{\alpha}(A)=\lim _{n \rightarrow \infty} \frac{1}{n^{\alpha}}|\{k \leq n: k \in A\}|
$$

in case this limit exists. Note that $\alpha$-density of any set reduces to its natural density in case $\alpha=1$. As we have earlier seen, the relation $d(A)+d(\mathbb{N}-A)=1$ is no longer true if we replace natural density by $f$-density, same is the case if natural density is replaced by $\alpha$-density for $\alpha \in(0,1)$. Moreover, as in the case of $f$-density, $\alpha$-density of finite sets is also zero.

Remark 1.6 If $A$ has zero $\alpha$-density for some $\alpha \in(0,1]$, then it has zero natural density. But converse need not be true, in the sense that a set having zero natural density may have non-zero $\alpha$-density for some $\alpha \in(0,1)$. For example, if we take $A=\{1,4,9, \ldots\}$ then $d(A)=0$ but $d_{\alpha}(A)=\infty$ for any $\alpha \in\left(0, \frac{1}{2}\right)$.

Definition 1.7 [26] Let $0<\alpha \leq 1$. A number sequence $x=\left(x_{k}\right)$ is said to be statistically convergent of order $\alpha$ to $l$, or $S_{\alpha}$-convergent to $l$, if for each $\varepsilon>0$,

$$
\begin{aligned}
& d_{\alpha}\left(\left\{k \in \mathbb{N}:\left|x_{k}-l\right| \geq \varepsilon\right\}\right)=0, \\
& \text { i.e., } \lim _{n \rightarrow \infty} \frac{1}{n^{\alpha}}\left|\left\{k \leq n:\left|x_{k}-l\right| \geq \varepsilon\right\}\right|=0,
\end{aligned}
$$

and we write it as $S_{\alpha}-\lim x_{k}=l$. The set of all statistically convergent sequences of order $\alpha$ is denoted by $S_{\alpha}$. In case $\alpha=1$, the statistical convergence of order $\alpha$ reduces to the statistical convergence. 
Throughout this paper $s, l_{\infty}$ and $c$ will denote the space of all, bounded and convergent sequences of complex numbers, respectively, and $\mathbb{C}$ will denote the set of all complex numbers. Moreover, we shall be concerned only with the sequences of scalars.

Spaces of strongly summable sequences were discussed by Kuttner [32], Maddox [33, 34] and others. The well-known spaces $w_{o}, w$ and $w_{\infty}$ of strongly Cesàro summable sequences are defined as:

$$
\begin{aligned}
& w_{o}=\left\{x \in s: \lim _{n \rightarrow \infty} \frac{1}{n} \sum_{k=1}^{n}\left|x_{k}\right|=0\right\}, \\
& w=\left\{x \in s: \lim _{n \rightarrow \infty} \frac{1}{n} \sum_{k=1}^{n}\left|x_{k}-l\right|=0 \text { for some number } l\right\}, \\
& w_{\infty}=\left\{x \in s: \sup _{n} \frac{1}{n} \sum_{k=1}^{n}\left|x_{k}\right|<\infty\right\} .
\end{aligned}
$$

Maddox [35] extended the definition of strong Cesàro summability to that of strong Cesàro summability with respect to a modulus and, consequently, introduced and studied the sequence spaces $w_{o}(f), w(f)$ and $w_{\infty}(f)$ which generalized the classical sequence spaces $w_{o}, w$ and $w_{\infty}$, respectively. Recently, Çolak [26] extended the definition of strong Cesàro summability to that of strong $p$-Cesàro summability of order $\alpha$, where $0<\alpha \leq 1$ and $p$ is a positive real number. The space of strongly $p$-Cesàro summable sequences of order $\alpha$ is denoted by $w_{p}^{\alpha}$.

In this paper, we first extend the notion of $\alpha$-density to that of $f_{\alpha}$-density in the same way as natural density was extended to $f$-density by Aizpuru et al. [24] and then introduce a new and more general nonmatrix summability method, namely $f$-statistical convergence of order $\alpha$, where $f$ is an unbounded modulus function and $\alpha$ is a real number such that $0<\alpha \leq 1$. The set of all $f$-statistically convergent sequences of order $\alpha$ is denoted by $S_{\alpha}^{f}$. In the second section, we establish inclusion relations among the set of all $f$-statistically convergent sequences of order $\alpha$ for different values of $\alpha$ and, in particular, we get an inclusion relation between the set of $f$-statistically convergent sequences of order $\alpha$ and the set of $f$-statistically convergent sequences. We also study inclusion relations between the newly introduced space $S_{\alpha}^{f}$ and the already existing spaces $S_{\alpha}$ and $S$. In the third section, we extend the notion of strong Cesàro summability of order $\alpha$ to that of strong Cesàro summability of order $\alpha(\alpha>0)$ with respect to a modulus $f$, in the same way as the notion of strong Cesàro summability was extended to that of strong Cesàro summability with respect to a modulus $f$ by Maddox [35] and, consequently, we obtain the sequence spaces $w_{\alpha, o}^{f}, w_{\alpha}^{f}$ and $w_{\alpha, \infty}^{f}$ which generalize and unify the corresponding earlier spaces of Maddox [35] and Çolak [26]. We establish inclusion relations between the newly introduced spaces $w_{\alpha, o}^{f}, w_{\alpha}^{f}, w_{\alpha, \infty}^{f}$ and finally obtain a condition under which the notions of strong Cesàro summability of order $\alpha$ with respect to a modulus $f$ and strong Cesàro summability of order $\alpha$ are equivalent. Some information on multipliers for $w_{\alpha, \infty}^{f}$ is also given. In the last section, we study inclusion relations between the space $S_{\alpha}^{f}$ of all $f$-statistically convergent sequences of order $\alpha$ and the space $w_{\alpha}^{f}$ of all strongly Cesàro summable sequences of order $\alpha$ with respect to a modulus $f$.

\section{$2 f$-Statistical convergence of order $\alpha$}

We begin this section by introducing a new concept of $f_{\alpha}$-density of a subset of $\mathbb{N}$. 
Definition 2.1 Let $f$ be an unbounded modulus function and $\alpha$ be any real number such that $0<\alpha \leq 1$. We define the $f_{\alpha}$-density of the subset $A$ of $\mathbb{N}$ by

$$
d_{\alpha}^{f}(A)=\lim _{n \rightarrow \infty} \frac{1}{f\left(n^{\alpha}\right)} f(|\{k \leq n: k \in A\}|)
$$

in case this limit exists, where $|\{k \leq n: k \in A\}|$ denotes the number of elements of $A$ not exceeding $n$.

Remark 2.2 For $\alpha=1$ and $f(x)=x$, the $f_{\alpha}$-density reduces to the natural density and when $\alpha=1, f_{\alpha}$-density becomes the $f$-density. In case $f(x)=x, f_{\alpha}$-density is the $\alpha$-density.

The equality $d_{\alpha}^{f}(A)+d_{\alpha}^{f}(\mathbb{N}-A)=1$ does not hold, in general, where $\alpha \in(0,1]$ and $f$ is any unbounded modulus. For example, if we take $f(x)=x^{p}, 0<p \leq 1, \alpha \in(0,1)$ and $A=\{2 n: n \in \mathbb{N}\}$, then $d_{\alpha}^{f}(A)=\infty=d_{\alpha}^{f}(\mathbb{N}-A)$.

As expected, finite sets have zero $f_{\alpha}$-density for any unbounded modulus $f$ and $\alpha \in(0,1]$.

Remark 2.3 For any unbounded modulus $f$ and $\alpha \in(0,1]$, if $d_{\alpha}^{f}(A)=0$ then, by the definition of limit and the fact that $f$ being modulus is subadditive, for every $p \in \mathbb{N}$, there exists $n_{o} \in \mathbb{N}$ such that for $n \geq n_{o}$ we have

$$
f(|\{k \leq n: k \in A\}|) \leq \frac{1}{p} f\left(n^{\alpha}\right) \leq \frac{1}{p} p f\left(\frac{n^{\alpha}}{p}\right)=f\left(\frac{n^{\alpha}}{p}\right)
$$

and since $f$ is increasing, we have

$$
\frac{1}{n^{\alpha}}|\{k \leq n: k \in A\}| \leq \frac{1}{p} \quad \text { and so } \quad d_{\alpha}(A)=0 .
$$

In view of Remark 2.3 and Remark 1.6, we have the following.

Remark 2.4 If $A \subset \mathbb{N}$ has zero $f_{\alpha}$-density for some unbounded modulus $f$ and for some $\alpha \in(0,1]$, then it has zero $\alpha$-density and hence zero natural density.

Remark 2.5 Converse of Remark 2.3 need not be true in the sense that a set having zero $\alpha$-density for some $\alpha \in(0,1]$ may have non-zero $f_{\alpha}$-density for some unbounded modulus $f$ with the same $\alpha$. Similarly, a set having zero natural density may have non-zero $f_{\alpha}$-density for some unbounded modulus $f$ and $\alpha \in(0,1]$. This can be verified by the following example.

Example 2.6 Let $f(x)=\log (x+1)$ and $A=\{1,4,9, \ldots\}=$ the set of squares of natural numbers. Then $d(A)=0$ and $d_{\alpha}(A)=0$ for $\alpha \in\left(\frac{1}{2}, 1\right]$ but $d_{\alpha}^{f}(A) \geq d^{f}(A)=1 / 2$. Therefore $d_{\alpha}^{f}(A) \neq 0$.

Lemma 2.7 For any unbounded modulus $f$ and $A \subset \mathbb{N}, d_{\beta}^{f}(A) \leq d_{\alpha}^{f}(A)$ for $0<\alpha \leq \beta \leq 1$.

Thus, for any unbounded modulus $f$ and $0<\alpha \leq \beta \leq 1$, if $A$ has zero $f_{\alpha}$-density then it has zero $f_{\beta}$-density. In particular, a set having zero $f_{\alpha}$-density for some $\alpha \in(0,1]$ has zero $f$-density. However, the converse is not true as the following example demonstrates. 
Example 2.8 Let $f(x)=x^{p}$, where $0<p \leq 1$ and $A=\{1,4,9, \ldots\}$. It is easy to see that $d^{f}(A)=0$. But $d_{\alpha}^{f}(A) \neq 0$ for any $\alpha \in(0,1 / 2)$ since

$$
\begin{aligned}
\frac{f(|\{k \leq n: k \in A\}|)}{f\left(n^{\alpha}\right)} & \geq \frac{([\sqrt{n}])^{p}-1}{\left(n^{\alpha}\right)^{p}} \\
& =\frac{([\sqrt{n}])^{p}}{n^{\alpha p}}-\frac{1}{n^{\alpha p}} \\
& =\frac{([\sqrt{n}])^{p}}{(\sqrt{n})^{p}} \frac{(\sqrt{n})^{p}}{n^{\alpha p}}-\frac{1}{n^{\alpha p}} \\
& =\frac{([\sqrt{n}])^{p}}{(\sqrt{n})^{p}} \frac{1}{n^{p(\alpha-1 / 2)}}-\frac{1}{n^{\alpha p}}
\end{aligned}
$$

and hence, taking limit as $n \rightarrow \infty$ on both sides, we get $d_{\alpha}^{f}(A)=\infty$ as $\lim _{n \rightarrow \infty} \frac{\left([\sqrt{n})^{p}\right.}{(\sqrt{n})^{p}}$ is finite.

We now introduce a new concept of $f$-statistical convergence of order $\alpha$ as follows.

Definition 2.9 Let $f$ be an unbounded modulus and $0<\alpha \leq 1$. A sequence $x=\left(x_{k}\right)$ is said to be $f$-statistically convergent of order $\alpha$ to $l$ or $S_{\alpha}^{f}$-convergent to $l$ if for each $\varepsilon>0$,

$$
\begin{aligned}
& d_{\alpha}^{f}\left(\left\{k \in \mathbb{N}:\left|x_{k}-l\right| \geq \varepsilon\right\}\right)=0, \\
& \quad \text { i.e., } \lim _{n \rightarrow \infty} \frac{1}{f\left(n^{\alpha}\right)} f\left(\left|\left\{k \leq n:\left|x_{k}-l\right| \geq \varepsilon\right\}\right|\right)=0 .
\end{aligned}
$$

In this case, we write $S_{\alpha}^{f}-\lim x_{k}=l$. The set of all sequences which are $f$-statistically convergent of order $\alpha$ is denoted by $S_{\alpha}^{f}$. We write $S_{\alpha, 0}^{f}$ to denote the set of all $f$-statistically null sequences of order $\alpha$. It is clear that $S_{\alpha, 0}^{f} \subset S_{\alpha}^{f}$ for any unbounded modulus $f$ and $\alpha \in(0,1]$.

On specifying $f$ and $\alpha$ in $S_{\alpha}^{f}$-convergence, we obtain some well-known nonmatrix convergence methods.

For $\alpha=1, S_{\alpha}^{f}$-convergence is the same as $f$-statistical convergence.

For $f(x)=x, S_{\alpha}^{f}$-convergence becomes statistical convergence of order $\alpha$.

For $f(x)=x$ and $\alpha=1, S_{\alpha}^{f}$-convergence reduces to statistical convergence.

Remark 2.10 In Definition 2.1, and hence in Definition 2.9 also, we have not allowed $\alpha$ to exceed 1 , since in case $\alpha>1$, the $f$-statistical limit of order $\alpha$ may not be unique as is clear from the following example.

Example 2.11 Let $f$ be an unbounded modulus such that $\lim _{t \rightarrow \infty} \frac{f(t)}{t}>0$. The existence of such a modulus is guaranteed, as Maddox [16] proved that for any modulus $f, \lim _{t \rightarrow \infty} \frac{f(t)}{t}$ exists.

Let $x=\left(x_{k}\right)$ be a sequence defined as follows:

$$
x_{k}=\left\{\begin{array}{ll}
1 & \text { if } k=2 n, \\
0 & \text { if } k \neq 2 n,
\end{array} \quad n=1,2,3, \ldots\right.
$$


Then we have

$$
\frac{1}{f\left(n^{\alpha}\right)} f\left(\left|\left\{k \leq n:\left|x_{k}-1\right| \geq \varepsilon\right\}\right|\right) \leq \frac{f\left(\frac{n}{2}\right)}{f\left(n^{\alpha}\right)}
$$

and

$$
\frac{1}{f\left(n^{\alpha}\right)} f\left(\left|\left\{k \leq n:\left|x_{k}-0\right| \geq \varepsilon\right\}\right|\right) \leq \frac{f\left(\frac{n}{2}\right)}{f\left(n^{\alpha}\right)} .
$$

Since $\lim _{t \rightarrow \infty} \frac{f(t)}{t}>0$, we have

$$
\lim _{n \rightarrow \infty} \frac{1}{f\left(n^{\alpha}\right)} f\left(\left|\left\{k \leq n:\left|x_{k}-1\right| \geq \varepsilon\right\}\right|\right)=0
$$

and

$$
\lim _{n \rightarrow \infty} \frac{1}{f\left(n^{\alpha}\right)} f\left(\left|\left\{k \leq n:\left|x_{k}-0\right| \geq \varepsilon\right\}\right|\right)=0
$$

for $\alpha>1$ and for each $\varepsilon>0$. Hence $x=\left(x_{k}\right)$ is $S_{\alpha}^{f}$-convergent to both 1 and 0, i.e., $S_{\alpha}^{f}-\lim x_{k}=$ 1 as well as $S_{\alpha}^{f}-\lim x_{k}=0$.

It is easy to see that every convergent sequence is $S_{\alpha}^{f}$-convergent, i.e., $c \subset S_{\alpha}^{f}$, for any unbounded modulus $f$ and $\alpha \in(0,1]$. But, as is clear from the following example, the converse need not hold always good.

Example 2.12 Consider the sequence $x=\left(x_{k}\right)$ defined by

$$
x_{k}=\left\{\begin{array}{ll}
1 & \text { if } k=n^{2}, \\
0 & \text { if } k \neq n^{2},
\end{array} \quad n=1,2,3, \ldots\right.
$$

and take $f(x)=x^{p}, 0<p \leq 1$. Then the sequence $x=\left(x_{k}\right) \in S_{\alpha}^{f}$ for $\alpha \in\left(\frac{1}{2}, 1\right]$ although it is not convergent.

Theorem 2.13 Let $f$ be an unbounded modulus and $0<\alpha \leq 1$. Let $x=\left(x_{k}\right), y=\left(y_{k}\right)$ be any two sequences. Then

(i) If $S_{\alpha}^{f}-\lim x_{k}=l$ and $c \in \mathbb{C}$, then $S_{\alpha}^{f}-\lim c x_{k}=c l$.

(ii) If $S_{\alpha}^{f}-\lim x_{k}=l_{1}$ and $S_{\alpha}^{f}-\lim y_{k}=l_{2}$, then $S_{\alpha}^{f}-\lim \left(x_{k}+y_{k}\right)=l_{1}+l_{2}$.

Theorem 2.14 Let $f$ be an unbounded modulus and $\alpha, \beta$ be such that $0<\alpha \leq \beta \leq 1$. Then $S_{\alpha}^{f} \subset S_{\beta}^{f}$ and the strict inclusion may occur.

Proof The inclusion follows readily in view of the fact that $f$ is increasing and $0<\alpha \leq \beta \leq$ 1. To show that the strict inclusion may occur, consider the sequence $x=\left(x_{k}\right)$ defined by

$$
x_{k}=\left\{\begin{array}{ll}
1 & \text { if } k=n^{3}, \\
0 & \text { if } k \neq n^{3},
\end{array} \quad n=1,2,3, \ldots\right.
$$

and take $f(x)=x^{p}, 0<p \leq 1$. Then $x \in S_{\beta}^{f}$ for $\beta \in\left(\frac{1}{3}, 1\right]$, but $x \notin S_{\alpha}^{f}$ for $\alpha \in\left(0, \frac{1}{3}\right)$. 
Corollary 2.15 If a sequence is $f$-statistically convergent of order $\alpha$ to l for some unbounded modulus $f$ and $0<\alpha \leq 1$, then it is $f$-statistically convergent to l, i.e., $S_{\alpha}^{f} \subset S^{f}$ and the strict inclusion may occur.

In view of Remarks 2.3 and 2.4 we have the following.

Theorem 2.16 Let $f$ be an unbounded modulus and $0<\alpha \leq 1$. Then

(i) $S_{\alpha}^{f} \subset S_{\alpha}$ and the strict inclusion may occur.

(ii) $S_{\alpha}^{f} \subset S$ and the strict inclusion may occur.

Proof To show that the strict inclusion may occur, consider the sequence $x=\left(x_{k}\right)$ defined as follows:

$$
x_{k}=\left\{\begin{array}{ll}
k & \text { if } k=n^{2}, \\
0 & \text { if } k \neq n^{2},
\end{array} \quad n=1,2,3, \ldots\right.
$$

Let $f(x)=\log (x+1)$. Then $x \in S_{\alpha}$ for $\alpha \in\left(\frac{1}{2}, 1\right]$ and hence $x \in S$. But $x \notin S_{\alpha}^{f}$ as $d_{\alpha}^{f}(\{k \in \mathbb{N}$ : $\left.\left.\left|x_{k}-0\right| \geq \varepsilon\right\}\right) \geq d^{f}\left(\left\{k \in \mathbb{N}:\left|x_{k}-0\right| \geq \varepsilon\right\}\right)=\frac{1}{2}(\neq 0)$.

To summarize, the overall picture regarding inclusions among the already existing spaces $c, S, S_{\alpha}, S^{f}$ and the newly introduced space $S_{\alpha}^{f}$ is as shown below:

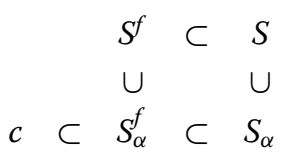

\section{Strong Cesàro summability of order $\alpha$ with respect to a modulus}

We begin this section by introducing the generalizations of the spaces of strongly Cesàro summable sequences of order $\alpha$.

Definition 3.1 Let $f$ be a modulus and $\alpha$ be a positive real number. We define

$$
\begin{aligned}
& w_{\alpha, o}^{f}=\left\{x \in s: \lim _{n \rightarrow \infty} \frac{1}{n^{\alpha}} \sum_{k=1}^{n} f\left(\left|x_{k}\right|\right)=0\right\}, \\
& w_{\alpha}^{f}=\left\{x \in s: \lim _{n \rightarrow \infty} \frac{1}{n^{\alpha}} \sum_{k=1}^{n} f\left(\left|x_{k}-l\right|\right)=0 \text { for some number } l\right\}, \\
& w_{\alpha, \infty}^{f}=\left\{x \in s: \sup _{n} \frac{1}{n^{\alpha}} \sum_{k=1}^{n} f\left(\left|x_{k}\right|\right)<\infty\right\} .
\end{aligned}
$$

Some well-known spaces are obtained by specializing $f$ and $\alpha$.

For example, if $\alpha=1$ then the sequence spaces defined above become $w_{o}(f), w(f)$ and $w_{\infty}(f)$ of Maddox [35], respectively.

If we take $f(x)=x$, then the first two spaces are the same as the spaces $w_{p}^{\alpha}$ and $w_{o p}^{\alpha}$ of Çolak [26] for $p=1$. In case $f(x)=x$, we shall denote the space $w_{\alpha, \infty}^{f}$ by $w_{\alpha, \infty}$. 
As a final illustration taking $f(x)=x$ and $\alpha=1$, we obtain the familiar spaces $w_{o}, w$ and $w_{\infty}$ of strongly Cesàro summable sequences, respectively.

Remark 3.2 In the spaces $w_{p}^{\alpha}$ and $w_{o p}^{\alpha}$ of Çolak [26], $\alpha$ is a positive real number less than or equal to 1 , whereas in our spaces $w_{\alpha, o}^{f}$ and $w_{\alpha}^{f}, \alpha$ is any positive real, i.e., there is no upper ceiling of $\alpha$.

Moreover, we have used the symbols $w_{o}^{f}, w^{f}, w_{\infty}^{f}, w_{\alpha, o}$ and $w_{\alpha}$ in place of $w_{o}(f), w(f)$, $w_{\infty}(f), w_{o p}^{\alpha}($ for $p=1)$ and $w_{p}^{\alpha}$ (for $p=1$ ), respectively, for the sake of notational convenience.

It is easy to see that $w_{\alpha, o}^{f}, w_{\alpha}^{f}$ and $w_{\alpha, \infty}^{f}$ are linear spaces over the complex field $\mathbb{C}$.

We now establish some inclusion relations between the spaces $w_{\alpha, o}^{f}, w_{\alpha}^{f}$ and $w_{\alpha, \infty}^{f}$.

\section{Theorem 3.3}

(i) For any modulus $f$ and positive $\alpha, w_{\alpha, o}^{f} \subset w_{\alpha, \infty}^{f}$.

(ii) For any modulus $f$ and $\alpha \geq 1, w_{\alpha}^{f} \subset w_{\alpha, \infty}^{f}$.

Proof We establish only the second inclusion, the first being obvious. Let $x \in w_{\alpha}^{f}$. By definition of modulus function (iii) and (ii), we have

$$
\frac{1}{n^{\alpha}} \sum_{k=1}^{n} f\left(\left|x_{k}\right|\right) \leq \frac{1}{n^{\alpha}} \sum_{k=1}^{n} f\left(\left|x_{k}-l\right|\right)+f(|l|) \frac{1}{n^{\alpha}} \sum_{k=1}^{n} 1
$$

and since $\alpha \geq 1$ and $x \in w_{\alpha}^{f}$, we have $x \in w_{\alpha, \infty}^{f}$, which completes the proof.

Our next theorem is a generalization of Theorem 4 of Maddox [35].

Theorem 3.4 For any modulus $f$ and $\alpha \geq 1$, we have $w_{\alpha} \subset w_{\alpha}^{f}, w_{\alpha, o} \subset w_{\alpha, o}^{f}$ and $w_{\alpha, \infty} \subset$ $w_{\alpha, \infty}^{f}$.

Proof The first two inclusions are easily proved, so we consider only the last inclusion. Let $x \in w_{\alpha, \infty}$ so that

$$
\sup _{n} \frac{1}{n^{\alpha}} \sum_{k=1}^{n}\left|x_{k}\right|<\infty .
$$

Let $\varepsilon>0$ and choose $\delta$ with $0<\delta<1$ such that $f(t)<\varepsilon$ for $0<t \leq \delta$. Consider $\frac{1}{n^{\alpha}} \sum_{k=1}^{n} f\left(\left|x_{k}\right|\right)=\sum_{1}+\sum_{2}$, where the first summation is over $\left|x_{k}\right| \leq \delta$ and the second is over $\left|x_{k}\right|>\delta$. Then $\sum_{1} \leq \varepsilon \frac{1}{n^{\alpha-1}}$, and for $\left|x_{k}\right|>\delta$ we use the fact that

$$
\left|x_{k}\right|<\left|x_{k}\right| / \delta<1+\left[\left|x_{k}\right| / \delta\right]
$$

where $[t]$ denotes the integral part of $t$. By definition of modulus function (iii) and (ii), we have, for $\left|x_{k}\right|>\delta$,

$$
f\left(\left|x_{k}\right|\right) \leq\left(1+\left[\left|x_{k}\right| / \delta\right]\right) f(1) \leq 2 f(1)\left|x_{k}\right| / \delta .
$$


Hence $\sum_{2} \leq 2 f(1) \delta^{-1} \frac{1}{n^{\alpha}} \sum_{k=1}^{n}\left|x_{k}\right|$, which together with $\sum_{1} \leq \varepsilon \frac{1}{n^{\alpha-1}}$ yields

$$
\frac{1}{n^{\alpha}} \sum_{k=1}^{n} f\left(\left|x_{k}\right|\right) \leq \varepsilon \frac{1}{n^{\alpha-1}}+2 f(1) \delta^{-1} \frac{1}{n^{\alpha}} \sum_{k=1}^{n}\left|x_{k}\right| .
$$

Since $\alpha \geq 1$ and $x \in w_{\alpha, \infty}$, we have $x \in w_{\alpha, \infty}^{f}$ and the proof is complete.

Theorem 3.5 Let $f$ be a modulus and $\alpha$ be a positive real number. If $\lim _{t \rightarrow \infty} \frac{f(t)}{t}>0$, then $w_{\alpha}^{f} \subset w_{\alpha}$.

Proof Following the proof of Proposition 1 of Maddox [16], we have $\beta=\lim _{t \rightarrow \infty} \frac{f(t)}{t}=$ $\inf \{f(t) / t ; t>0\}$. By definition of $\beta$, we have $f(t) \geq \beta t$ for all $t \geq 0$. Since $\beta>0$, we have $t \leq \beta^{-1} f(t)$ for all $t \geq 0$ and so

$$
\frac{1}{n^{\alpha}} \sum_{k=1}^{n}\left|x_{k}-l\right| \leq \beta^{-1} \frac{1}{n^{\alpha}} \sum_{k=1}^{n} f\left(\left|x_{k}-l\right|\right)
$$

from where it follows that $x \in w_{\alpha}$ whenever $x \in w_{\alpha}^{f}$.

Remark 3.6 We have just seen in Theorem 3.4 that the inclusion $w_{\alpha} \subset w_{\alpha}^{f}$ is true in case $\alpha \geq 1$, without any restriction on the modulus $f$, whereas in Theorem 3.5 we have established that $w_{\alpha}^{f} \subset w_{\alpha}$ is true only for such a modulus function $f$ for which $\lim _{t \rightarrow \infty} \frac{f(t)}{t}>0$, there being no restriction on $\alpha$.

Combining these two results we have the following.

Theorem 3.7 Let $f$ be any modulus such that $\lim _{t \rightarrow \infty} \frac{f(t)}{t}>0$ and $\alpha \geq 1$. Then $w_{\alpha}^{f}=w_{\alpha}$.

Theorem 3.8 Let $f$ be a modulus and $\beta \geq \alpha>0$. Then $w_{\alpha}^{f} \subset w_{\beta}^{f}$ and the strict inclusion may occur.

Proof To show that the strict inclusion may occur, let $f$ be a modulus and consider the sequence $x=\left(x_{k}\right)$ defined by

$$
x_{k}=\left\{\begin{array}{ll}
1 & \text { if } k=n^{2}, \\
0 & \text { if } k \neq n^{2},
\end{array} \quad n=1,2,3, \ldots\right.
$$

Using the fact that $f(0)=0$, it is easy to see that

$$
\frac{1}{n^{\beta}} \sum_{k=1}^{n} f\left(\left|x_{k}-0\right|\right) \leq \frac{\sqrt{n}}{n^{\beta}} f(1)=\frac{1}{n^{\beta-1 / 2}} f(1) \quad \text { for every } n \in \mathbb{N} \text {. }
$$

Since $\frac{1}{n^{\beta-1 / 2}} f(1) \rightarrow 0$ as $n \rightarrow \infty$ for $\beta>1 / 2$, so $x \in w_{\beta}^{f}$ for $\beta>1 / 2$. Also

$$
\frac{1}{n^{\alpha}} \sum_{k=1}^{n} f\left(\left|x_{k}-0\right|\right) \geq \frac{\sqrt{n}-1}{n^{\alpha}} f(1) \quad \text { for every } n \in \mathbb{N}
$$


and $\frac{\sqrt{n}-1}{n^{\alpha}} f(1) \rightarrow \infty$ as $n \rightarrow \infty$ for $0<\alpha<1 / 2$, which implies that $x \notin w_{\alpha}^{f}$ for $0<\alpha<$ $1 / 2$.

Some information on multipliers for $w_{\alpha, \infty}^{f}$ is given below. For any set $E$ of sequences, the space of multipliers of $E$, denoted by $M(E)$, is given by

$$
M(E)=\{a \in s: a x \in E \text { for all } x \in E\} .
$$

Theorem 3.9 Let $f$ be a modulus and $\alpha$ be a positive real number. Then

(i) $l_{\infty} \subset M\left(w_{\alpha, \infty}^{f}\right)$,

(ii) $M\left(w_{\alpha, \infty}^{f}\right) \subset w_{\alpha, \infty}^{f}$ if $\alpha \geq 1$,

(iii) If $f$ is bounded and $\alpha \geq 1$, then

$$
M\left(w_{\alpha, \infty}^{f}\right)=w_{\alpha, \infty}^{f}=s
$$

Proof (i) $b \in l_{\infty}$ implies that $\left|b_{k}\right|<1+[H]$ for some $H>0$ and for all $k$, and so

$$
\frac{1}{n^{\alpha}} \sum_{k=1}^{n} f\left(\left|b_{k} x_{k}\right|\right) \leq(1+[H]) \frac{1}{n^{\alpha}} \sum_{k=1}^{n} f\left(\left|x_{k}\right|\right) \quad \text { for all } x \in w_{\alpha, \infty}^{f}
$$

which gives the first inclusion.

(ii) The second inclusion follows from the fact that $e=(1,1,1, \ldots) \in w_{\alpha, \infty}^{f}$ for $\alpha \geq 1$.

(iii) If $f$ is bounded and $\alpha \geq 1$, then for any $x=\left(x_{k}\right) \in s$,

$$
\frac{1}{n^{\alpha}} \sum_{k=1}^{n} f\left(\left|x_{k}\right|\right) \leq \sup \{f(y): y \geq 0\} \frac{1}{n^{\alpha-1}}
$$

so that $w_{\alpha, \infty}^{f}=s$. Similarly, by part (ii) and the above argument, we have $M\left(w_{\alpha, \infty}^{f}\right)=w_{\alpha, \infty}^{f}$.

Recall [36] that a sequence space $E$ is called

1. normal if $y=\left(y_{k}\right) \in E$ whenever $\left|y_{k}\right| \leq\left|x_{k}\right|, k \geq 1$, for some $x=\left(x_{k}\right) \in E$,

2. monotone if it contains the canonical preimages of all its stepspaces.

Theorem 3.10 [37] If E is a sequence space, the following are equivalent.

(i) $E$ is normal,

(ii) $l_{\infty} \subset M(E)$,

(iii) $M(E)$ is normal.

Lemma 3.11 [36] Every normal sequence space is monotone.

In view of Theorems 3.9, 3.10 and Lemma 3.11, we have the following.

Theorem 3.12 For any modulus $f$ and positive real number $\alpha$, the spaces $w_{\alpha, \infty}^{f}$ and $M\left(w_{\alpha, \infty}^{f}\right)$ are normal as well as monotone. 


\section{Relation between $f$-statistical convergence of order $\alpha$ and strong Cesàro summability of order $\alpha$ with respect to a modulus}

It was shown by Çolak [26] that for $\alpha \in(0,1]$, if a sequence is strongly Cesàro summable of order $\alpha$ to $l$, then it is statistically convergent of order $\alpha$ to $l$. We now wish to find some condition on $f$, if any, so that strong Cesàro summability of order $\alpha$ with respect to a modulus $f$ to $l$ of a sequence implies its $f$-statistical convergence of order $\alpha$ to $l$. We also wish to obtain some condition on $f$, if any, under which strong Cesàro summability of order $\alpha$ with respect to a modulus $f$ to $l$ of a sequence implies its statistical convergence of order $\alpha$ to $l$.

Maddox [35] showed the existence of an unbounded modulus $f$ for which there exists a positive constant $c$ such that $f(x y) \geq c f(x) f(y)$ for all $x \geq 0, y \geq 0$. Using this we have the following.

Theorem 4.1 Let $0<\alpha \leq \beta \leq 1$. Let $f$ be an unbounded modulus such that there is a positive constant $c$ such that $f(x y) \geq c f(x) f(y)$ for all $x \geq 0, y \geq 0$ and $\lim _{t \rightarrow \infty} \frac{f(t)}{t}>0$. If a sequence is strongly Cesàro summable of order $\alpha$ with respect to $f$ to $l$, then it is $f$-statistically convergent of order $\beta$ to $l$.

Proof For any sequence $x=\left(x_{k}\right)$ and $\varepsilon>0$, by the definition of modulus function (ii) and (iii), we have

$$
\begin{aligned}
\sum_{k=1}^{n} f\left(\left|x_{k}-l\right|\right) & \geq f\left(\sum_{k=1}^{n}\left|x_{k}-l\right|\right) \\
& \geq f\left(\left|\left\{k \leq n:\left|x_{k}-l\right| \geq \varepsilon\right\}\right| \varepsilon\right) \\
& \geq c f\left(\left|\left\{k \leq n:\left|x_{k}-l\right| \geq \varepsilon\right\}\right|\right) f(\varepsilon)
\end{aligned}
$$

and since $\alpha \leq \beta$,

$$
\begin{aligned}
\frac{1}{n^{\alpha}} \sum_{k=1}^{n} f\left(\left|x_{k}-l\right|\right) & \geq \frac{c f\left(\left|\left\{k \leq n:\left|x_{k}-l\right| \geq \varepsilon\right\}\right|\right) f(\varepsilon)}{n^{\alpha}} \\
& \geq \frac{c f\left(\left|\left\{k \leq n:\left|x_{k}-l\right| \geq \varepsilon\right\}\right|\right) f(\varepsilon)}{n^{\beta}} \\
& =\frac{c f\left(\left|\left\{k \leq n:\left|x_{k}-l\right| \geq \varepsilon\right\}\right|\right) f(\varepsilon) f\left(n^{\beta}\right)}{n^{\beta} f\left(n^{\beta}\right)} .
\end{aligned}
$$

From where, using the fact that $\lim _{t \rightarrow \infty} \frac{f(t)}{t}>0$ and $x \in w_{\alpha}^{f}$, it follows that $x \in S_{\beta}^{f}$ and the proof is complete.

If we take $\beta=\alpha$ in Theorem 4.1, we have the following.

Corollary 4.2 Let $f$ be an unbounded modulus such that there is a positive constant $c$ such that $f(x y) \geq c f(x) f(y)$ for all $x \geq 0, y \geq 0$ and $\lim _{t \rightarrow \infty} \frac{f(t)}{t}>0$. Let $0<\alpha \leq 1$. If a sequence is strongly Cesàro summable of order $\alpha$ with respect to $f$ to $l$, then it is $f$-statistically convergent of order $\alpha$ to $l$.

If we take $\alpha=1$ in Corollary 4.2, then we have the following. 
Corollary 4.3 Let $f$ be an unbounded modulus such that there is a positive constant $c$ such that $f(x y) \geq c f(x) f(y)$ for all $x \geq 0, y \geq 0$ and $\lim _{t \rightarrow \infty} \frac{f(t)}{t}>0$. If a sequence is strongly Cesàro summable with respect to $f$ to $l$, then it is $f$-statistically convergent to $l$.

Remark 4.4 If we take $f(x)=x$ in Theorem 4.1, we obtain Theorem 3.8 of Çolak [26] for the case $p=1$.

Theorem 4.5 Let $f$ be a modulus function such that $\lim _{t \rightarrow \infty} \frac{f(t)}{t}>0$ and $\alpha \in(0,1]$. If a sequence is strongly Cesàro summable of order $\alpha$ with respect to $f$ to $l$, then it is statistically convergent of order $\alpha$ to $l$.

Taking $\alpha=1$ in Theorem 4.5 we obtain the following result which is a particular case of part (a) of Theorem 8 of Connor [17].

Corollary 4.6 Let $f$ be a modulus function such that $\lim _{t \rightarrow \infty} \frac{f(t)}{t}>0$. If a sequence is strongly Cesàro summable with respect to $f$ to $l$, then it is statistically convergent to $l$.

Taking $f(x)=x$ and $\alpha=1$ in Theorem 4.5, we obtain the following result, which is contained in Theorem 2.1 of Connor [8], for the case $q=1$.

Corollary 4.7 If a sequence is strongly Cesàro summable to l, then it is statistically convergent to $l$.

Competing interests

The authors declare that they have no competing interests.

Authors' contributions

VKB and SD contributed equally. All authors read and approved the final manuscript.

\section{Author details}

${ }^{1}$ Department of Mathematics, Kurukshetra University, Kurukshetra, 136119, India. ${ }^{2}$ Department of Mathematics, KVA DAV College for Women, Karnal, 132001, India.

\section{Acknowledgements}

The authors wish to thank the referees for their valuable suggestions, which have improved the presentation of the paper Received: 17 July 2015 Accepted: 30 September 2015 Published online: 14 October 2015

\section{References}

1. Zygmund, A: Trigonometric Series, 2nd edn. Cambridge University Press, London (1979)

2. Steinhaus, H: Sur la convergence ordinaire et la convergence asymptotique. Colloq. Math. 2, 73-74 (1951)

3. Fast, H: Sur la convergence statistique. Colloq. Math. 2, 241-244 (1951)

4. Schoenberg, IJ: The integrability of certain functions and related summability methods. Am. Math. Mon. 66, 361-375 (1959)

5. Buck, RC: Generalized asymptotic density. Am. J. Math. 75, 335-346 (1953)

6. Šalát, T: On statistically convergent sequences of real numbers. Math. Slovaca 30(2), 139-150 (1980)

7. Fridy, JA: On statistical convergence. Analysis 5, 301-313 (1985)

8. Connor, JS: The statistical and strong $p$-Cesàro convergence of sequences. Analysis 8, 47-63 (1988)

9. Çinar, M, Karakaş, M, Et, M: On pointwise and uniform statistical convergence of order $\alpha$ for sequences of functions. Fixed Point Theory Appl. 2013, Article ID 33 (2013)

10. Et, M, Alotaibi, A, Mohiuddine, SA: On $\left(\Delta^{m}, \mathcal{I}\right)$-statistical convergence of order $\alpha$. Sci. World J. 2014, Article ID 535419 (2014). doi:10.1155/2014/535419

11. Savas, E: Double almost lacunary statistical convergence of order $\alpha$. Adv. Differ. Equ. 2013, Article ID 254 (2013)

12. Sengül, H, Et, M: On lacunary statistical convergence of order $\alpha$. Acta Math. Sci. 34(2), 473-482 (2014)

13. Niven, I, Zuckerman, HS: An Introduction to the Theory of Numbers, 4th edn. Wiley, New York (1980)

14. Nakano, H: Concave modulars. J. Math. Soc. Jpn. 5, 29-49 (1953)

15. Ruckle, WH: FK spaces in which the sequence of coordinate vectors is bounded. Can. J. Math. 25, $973-978$ (1973)

16. Maddox, IJ: Inclusion between FK spaces and Kuttner's theorem. Math. Proc. Camb. Philos. Soc. 101, $523-527$ (1987)

17. Connor, J: On strong matrix summability with respect to a modulus and statistical convergence. Can. Math. Bull. 32(2), 194-198 (1989) 
18. Ghosh, D, Srivastava, PD: On some vector valued sequence spaces defined using a modulus function. Indian J. Pure Appl. Math. 30(8), 819-826 (1999)

19. Bhardwaj, VK, Singh, N: On some sequence spaces defined by a modulus. Indian J. Pure Appl. Math. 30(8), 809-817 (1999)

20. Bhardwaj, VK, Singh, N: Some sequence spaces defined by $\left|\bar{N}, p_{n}\right|$ summability and a modulus function. Indian J. Pure Appl. Math. 32(12), 1789-1801 (2001)

21. Bhardwaj, VK, Singh, N: Banach space valued sequence spaces defined by a modulus. Indian J. Pure Appl. Math. 32(12), 1869-1882 (2001)

22. Çolak, R: Lacunary strong convergence of difference sequence spaces with respect to a modulus function. Filomat 17, 9-14 (2003)

23. Altin, Y, Et, M: Generalized difference sequence spaces defined by a modulus function in a locally convex space. Soochow J. Math. 31(2), 233-243 (2005)

24. Aizpuru, A, Listàn-Garcia, MC, Rambla-Barreno, F: Density by moduli and statistical convergence. Quaest. Math. 37, 525-530 (2014)

25. Bhardwaj, VK, Dhawan, S, Gupta, S:. Density by moduli and statistical boundedness (communicated)

26. Çolak, R: Statistical convergence of order $\alpha$. In: Modern Methods in Analysis and Its Applications, pp. 121-129. Anamaya Publishers, New Delhi (2010)

27. Bhunia, S, Das, P, Pal, S: Restricting statistical convergence. Acta Math. Hung. 134(1-2), 153-161 (2012)

28. Çolak, R, Bektaş, CA: $\lambda$-Statistical convergence of order $\alpha$. Acta Math. Sci. 31(3), $953-959$ (2011)

29. Das, P, Savas, E: On $\mathcal{I}$-statistical and $\mathcal{I}$-lacunary statistical convergence of order $\alpha$. Bull. Iran. Math. Soc. 40(2), 459-472 (2014)

30. Das, P, Ghosal, S, Som, S: Statistical convergence of order $\alpha$ in probability. Arab J. Math. Sci. (2014). doi:10.1016/j.ajmsc.2014.06.002

31. Et, M, Çinar, M, Karakaş, M: On $\lambda$-statistical convergence of order $\alpha$ of sequences of functions. J. Inequal. Appl. 2013 Article ID 204 (2013)

32. Kuttner, B: Note on strong summability. J. Lond. Math. Soc. 80(2), 118-122 (1946)

33. Maddox, IJ: Spaces of strongly summable sequences. Q. J. Math. 8(2), 345-355 (1967)

34. Maddox, IJ: On Kuttner's theorem. J. Lond. Math. Soc. 43, 285-290 (1968)

35. Maddox, IJ: Sequence spaces defined by a modulus. Math. Proc. Camb. Philos. Soc. 100, 161-166 (1986)

36. Kamthan, PK, Gupta, M: Sequence Spaces and Series. Dekker, New York (1981)

37. Rath, D: Multiplier sets and orthogonal duals of sequence spaces. J. Anal. 4, 23-33 (1996)

\section{Submit your manuscript to a SpringerOpen ${ }^{\circ}$ journal and benefit from:}

- Convenient online submission

Rigorous peer review

- Immediate publication on acceptance

- Open access: articles freely available online

- High visibility within the field

- Retaining the copyright to your article 